Physics, Chemistry, and Dynamics of Interplanetary Dust

ASP Conference Series, Vol. 104, 1996

Bo A. S. Gustafson and Martha S. Hanner (eds.)

\title{
Martian Self-Sustaining Dust Torus
}

\author{
Sho Sasaki \\ Geological Institute, University of Tokyo, Hongo, Tokyo 113. Japan
}

\begin{abstract}
Recent theoretical studies show that orbits of circummartian dust particles are controlled by solar radiation pressure and the Martian oblateness. Smaller dust particles $(<22 \mu \mathrm{m})$ with enhanced orbital eccentricity are quickly captured by Mars. We find that the collisions of ring particles with Phobos and Deimos are the most important dust sources. Erosion of Phobos should set the upper limit on the dust production efficiency controlling this self-sustaining mechanism, and then the ring dust abundance.
\end{abstract}

\section{Introduction}

Giant planets have dust rings whose major source is bombardments of interplanetary particles on small satellites. Mars also has small satellites Phobos and Deimos where ejecta may easily escape against gravity because of the low escape velocity $(\sim 10 \mathrm{~m} / \mathrm{s})$. Recently many works have been done on a Martian dust ring/torus whose particles originate from Phobos and Deimos (Soter 1971. Ip \& Banaszkiewicz 1990, Horányi et al. 1990, Juhász et al. 1993, Kholshevnikov et al. 1093, Ishimoto \& Mukai 1994, Hamilton 1996, Sasaki 1993 \& 1994, Juhász. \& Horányi 1995, Ishimoto 1996). However, there have been no direct measurements of dust particles around Mars yet. Analysis of Viking Orbiter image data suggested no dust rings whose optical depth is greater than $3 \times 10^{-5}$ (Duxbury \& Ocampo 1988). Only plasma measurement by the ion mass spectrometer ASPERA on PHOBOS-2 showed indirect evidence of a gas torus involving submicron dust at the orbit of Phobos orbit (Dubinin et al. 1990).

\section{Radiation Pressure and Martian Oblateness}

Around Mars, submicron particles are charged, scattered by solar wind magnetic field, and trapped by Mars quickly. Orbits of dust particles larger than one micron are affected by solar radiation pressure: dust orbital eccentricity around Mars oscillates with a period close to one Mars yrar The amplitude of the ec centricity oscillation $e_{\max }$ depends on the optical parameter $\beta$, ratio of radiation force to the solar gravity. Assuming a silicate grain with radius $r_{d}$ larger than a few micron, we have at Phobos' orbit $\epsilon_{\max }=0.3\left(\beta / 10^{-2}\right)=0.3\left(r_{d} / 20 \mu \mathrm{m}\right)^{-1}$ A small particle whose $e_{\max }$ becomes larger than $0.63\left(r_{d}<8 \mu \mathrm{m}\right.$ here $)$ collides with Mars in a year As for larger particles, collision with a satellite is the most important loss mechanism and their life time is much longer. Since the life time is proportional to the volume containing the dust $V$, particles with large $f_{m i x}$. i.e.. particles around $10 \mu \mathrm{m}$ would dominate around Mars (e.g.. Juhász et al. 1993. Ishimoto \& Mukai 1994). 
However, Martian oblateness. the $\mathrm{J}_{2}$ term of the gravitational potential. should shift the periapsis of an eccentric orbit. The oscillation period in eccentricity under the radiation pressure is prolonged and $e_{m a x}$ increases. At the orbit of Phobos, the phase shift can be so large at $r_{d} \leq 200 \mu \mathrm{m}$ that the resonance with the phase shift from the radiation pressure should enhance $\epsilon_{\max }$ significantly (Juhász and Horányi 199.5. Hamilton 1996. Ishimoto 1996). Particles escaping the trap by Mars should be larger $(22 \mu \mathrm{m})$ and the expected dust mumber density should be smaller Orbital inclination $i$ is kept small $(\sim 0.01 \mathrm{ra}$ dian) and dust distribution could be called a "ring"; smaller distribution volume would also result in shorter dust life times. and thus, smaller dust abundance.

At I) rimos' orbit, Martian oblateness with the radiation pressure should enhance the orbital inclination $i$ of particles so that the dust distribution becomes a "torus" (Ishimoto 1996, Hamilton 1996). The larger volume of Deimos dust "torus" should keep dust abundance high in comparison with Phobos" "ring"

\section{Self-Sustaining Mechanism}

Dust particles impacting onto satellites do not always lead to dust loss. Some fraction of the collisional ejecta may escape from the satellite so that the Martian dust ring/torus is self-sustained (Sasaki 1994). This mechanism was originally proposed for Saturn's E ring (Hamilton \& Burns 1994). We define the dust production efficiency $\eta$ as the total mass of ejecta whose velocity is higher than the escape velocity to the mass of the impacting particle. When a particle belonging to the Phobos ring with high $e$ hits Phobos, the collision velocity $v$ is as high as a few $100 \mathrm{~m} / \mathrm{s}-1 \mathrm{~km} / \mathrm{s}$, and when a particle of the Deimos torus with high $e$ hits Phobos, $v$ is a few $\mathrm{km} / \mathrm{s}$. The dust production efficiency is estimated from analytical scaling laws under gravity or strength regime. Although our estimate predicts a lower dust production at interplanetary particle collisions (high $v$ ) than that used in previous works, the efficiency $\eta$ is $5-10$ when a particle hits Phobos at $1 \mathrm{~km} / \mathrm{s}$ and slightly higher when a particle hits Deimos because of smaller escape velocity (Sasaki 1994). Ejecta particles should have a size distribution and we should adopt a smaller $\eta$ for producing the same-sized particles. If escaping cjecta have a power-law size distribution and the largest size is determined from the criterion that integrated particle number from the size to the infinity in one, the maximum size of ejecta particles can be as large as that of the incoming particle especially at collisions onto Phobos (Ishimoto 1996).

When the dust ring is self-sustained, a change of the dust size by dust-dust collision is the main dust loss mechanism. When dust size $r_{d}$ becomes smaller by collisional disruption, stronger radiation pressure enhances $e$ so that the particle collides with Mars. When the dust size becomes larger by coalescence ( $>200 \mu \mathrm{m}$ for the Phobos ring)., would be smaller and dust is readily consumed by satellites at relatively low $(\leq 100 \mathrm{~m} / \mathrm{s})$ collisional velocity which would not produce escaping secondary ejecta. Considering the balance between dust supply from the satellite and the loss by the dust-dust collisions, we have a mean number density of dust under the particle-in-a-box approximation:

$$
n_{d} \sim \frac{\eta-1}{V}\left(\frac{r_{s a t}}{r_{d}}\right)^{2}
$$




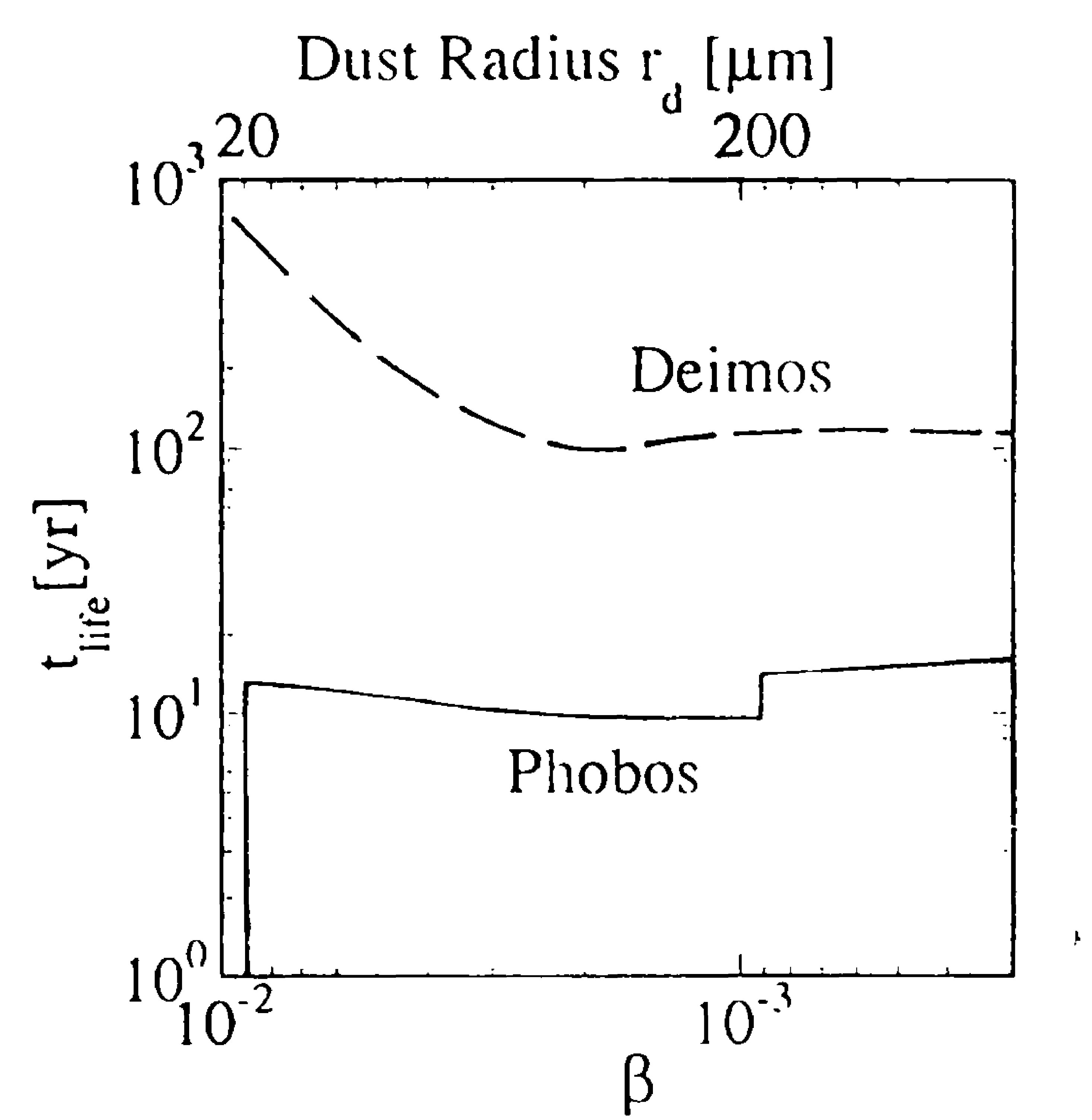

Fig. 1 Life time of dust particles from Phobos and Deimos. For Phobos $t=0.9 \mathrm{yr}$ at $r_{d}<22 \mu \mathrm{m}$.

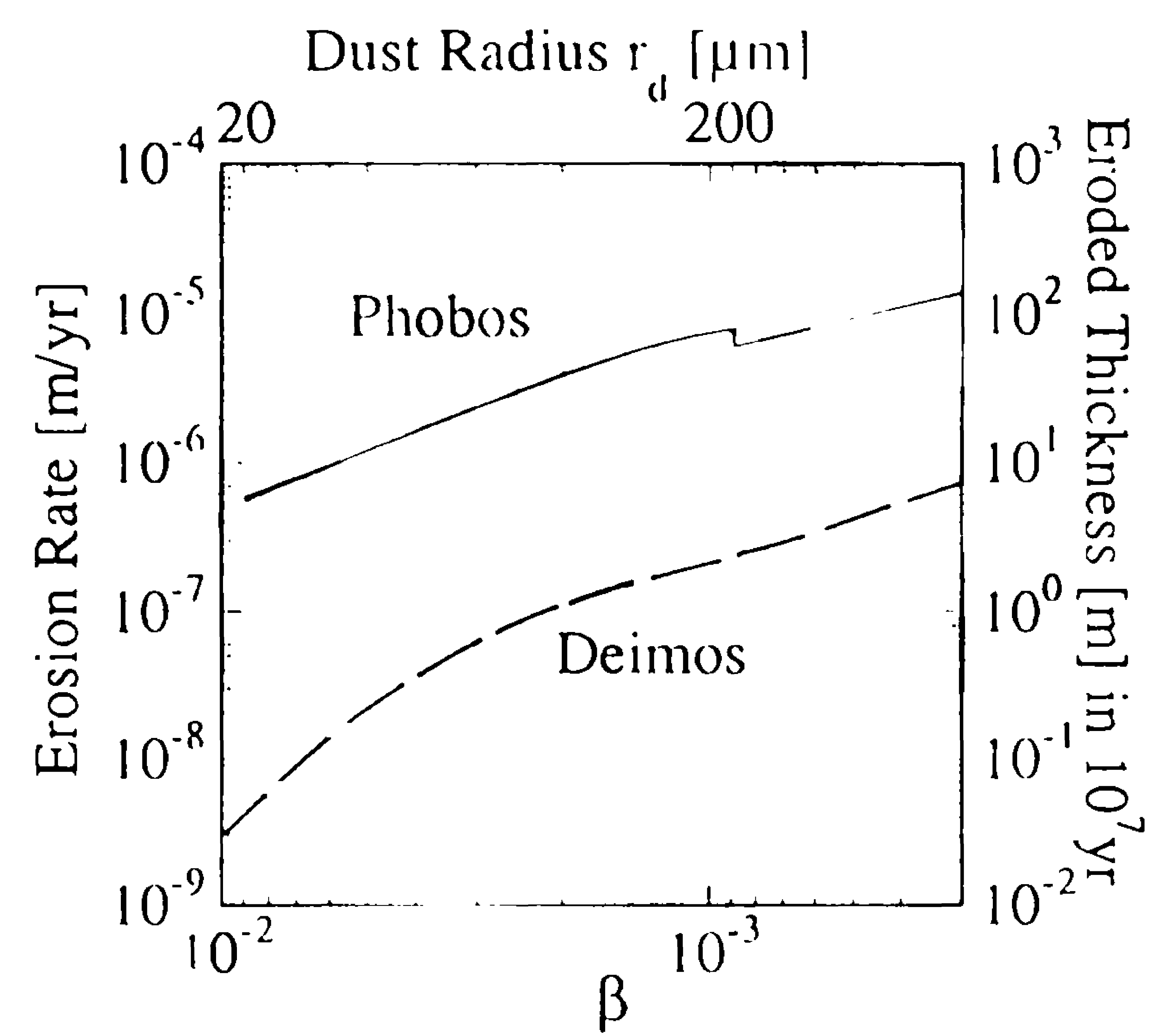

Fig. 2 Erosion rate of Phobos and Deimos by ring dust impacts when $\eta-1=1$.

where $V$ is the ring volume and $r_{\text {sat }}$ is the satellite radius. Then we have

$$
\begin{aligned}
& n_{d} V \sim 1.6 \times 10^{17}(\eta-1)\left(\frac{r_{d}}{30 \mu \mathrm{m}}\right)^{-2}-\text { for Phobos "ring" } \\
& n_{d} V \sim 4.9 \times 10^{17}(\eta-1)\left(\frac{r_{d}}{10 \mu \mathrm{m}}\right)^{-2}-\text { for Deimos "torus" }
\end{aligned}
$$

for the total particle number Thus the number density in the Phobos' ring with its smaller $V$ must be higher than that in the Deimos torus. As seen in Fig. 1, life time of particles from Deimos is longer than that of particles from Phobos.

From the data analysis of the Viking Thermal Infrared Mapper, typical grain sizes on Phobos' surface regolith is estimated to be $50-100 \mu \mathrm{m}$. When the dust impact velocity onto the satellite is not too high, the typical ejecta size can be represented by the regolith size. This size corresponds to the dust size of the self-sustaining ring where the eccentricity is enhanced by the Martian oblateness.

\section{Satellite Erosion}

Dust ejection is a mass loss mechanism for the satellite itself. Significant dust bombardments supplying ring particles would erode the satellite surface. We can estimate the surface erosion rate, i.e., the dust supply rate from the total number and life time of dust particles. Our results are shown in Fig. 2 when $\eta-1=1$. For Phobos, when the dust radius is larger than $40 \mu \mathrm{m}$, the surface erosion rate is larger than $10^{-6} \mathrm{~m} / \mathrm{yr}$, that is. larger than $10 \mathrm{~m}$ during the period $10^{7} \mathrm{yr}$. Note that the orbit of Phobos is now approaching Mars due to tidal interaction with Mars. Its residence time in the eccentricity enhancement zone (3.5R $R_{M}$ - to present $2.8 R_{M}$, Ishimoto 1996) is around a few $10^{7} y \mathrm{r}$ to $10^{8} \mathrm{yr}$ (Szeto, 
1983) and much smaller than the solar system age. However, because small-scale impact morphologies remain on the present Phobos surface, the surface erosion of Phobos would be less than $10 \mathrm{~m}$. This requires smaller dust production efficiency $\eta-1 \leq 0.1$ since the erosion rate is proportional to $\eta-1$.

On the other hand, the erosion rate of Deimos at $\eta-1=1$ is much smaller than that of Phobos; it is less than $10^{-8} \mathrm{~m} / \mathrm{yr}$ when the dust radius is larger than $30 \mu \mathrm{m}$. This is because the volume of Deimos' torus is much larger than that of Phobos' ring and the life time of Deimos' clust is longer than 100yr (Fig. 1). The dust supply rate can be much smaller than that for Phobos (Fig. 2).

\section{Optical Thickness of a Dust Ring and Its Detection}

When $\eta-1=0.1$, the optical thickness of the Phobos ring is $6 \times 10^{-8}$ along the direction perpendicular to the ring and $6 \times 10^{-6}(i / 0.01)^{-1}$ along the parallel direction. We have a smaller value for the Deimos torus $1 \times 10^{-7}(i / 0.2)^{-1}$ even if we use $\eta-1=1$. The above estimate is smaller than the upper limit by Viking Orbiter data $3 \times 10^{-5}$ Note that the dependence on particle size is cancelled out. If the self-sustained mechanism is operating, we may detect the Phobos' ring optically using more sensitive measurement in the future.

From 1999, the impact ionization dust detector MDC; on ISAS PLANET-B will directly measure dust particle impacts around Mars (Igenbergs et al. 1996). PLANET B will be on a retrograde elliptic orbit which crosses the above dust ring/torus. If ring particles are supplied by collisions of interplanetary particles onto satellites only, the dust abundance will be too small to be directly detected unless the dust ejection efficiency is high (Ishimoto \& Mukai 1994). Under the self-sustaining mechanism, however, MDC with aperture $140 \mathrm{~cm}^{2}$ will be able to detect a few particles with size $20-30 \mu \mathrm{m}$ at each passage through the ring.

\section{References.}

Dubinin, E. M. et al. 1990, Geophys. Res. Lett., 17, 861

Duxbury, T C. \& Ocampo, A. C. 1988, Icarus, 76, 160

Hamilton, D. P 1996, Icarus, in press.

Hamilton, D. P. \& Burns, J A. 1994, Science, 264, 550

Ilorányi, M. et al. 1990, Geophys. Res. Lett., 17, 853

Igenbergs. et al. 1996, in this volume.

Ip, W.-H. \& Banaszkiewicz, M. 1990, Geophys. Res. Lett. 17, 857

Ishimoto, H. 1996, Icarus, in press.

Ishimoto, H. \& Mukai, T 1994, Planet. Space. Sci., 42, 691

Juhász, A. \& Horányi, M. 1995, J Geophys. Res., 100, 3277

Juháš, A. et al. 1993, J Geophys. Res., 98, 1205

Kholshevnikov, K. V et al. 1993, Icarus, 105, 351

Lunine, J I. et al. 1982, J. Geophys. Res., 87, 10297

Sasaki, S 199:3. Proc. 26th ISAS Lunar Planet. Symp., 40

Sasaki, S. 199.4, Proc. 27th ISAS Lunar Planet. Symp.. 47

Soter, S 1971. Cornell Center Radliophys. \& Space Res. Rep. 462.

Szeto, A. M. K. 1983, Icarus, 55, 133 